\title{
EFFECT OF AUTOGENOUS BONE MARROW MESENCHYMAL STEM CELLS IN HEALING OF MANDIBULAR OSSEOUS DEFECTS GRAFTED WITH BETA TRICALCIUM PHOSPHATE IN DOGS
}

\author{
Amira S. Eissa ${ }^{1} M S C$, Sahar Sh. Karam ${ }^{2} P h D$, Samia S. Omar ${ }^{3} P h D$, Mohamed I. Sayed ${ }^{4} P h D$
}

\begin{abstract}
INTRODUCTION: Bone defect above critical size does not heal completely by itself and thus represents major clinical challenge to reconstructive surgery. Tissue engineering is a new approach for the repair of osseous defects. $\beta$ tricalcium phosphate ( $\beta$-TCP) loaded with autogenous bone marrow derived mesenchymal stem cells (BMSCs) is suggested to have a promising osteogenic characteristics that support its potential in tissue engineering to repair bone defects.

OBJECTIVES: was to evaluate the efficacy of autogenous (BMSCs) by its transplantation into local defects using ( $\beta$-TCP) as a carrier.

MATERIALS AND METHODS: Ten male Mongrel dogs weighing $25 \mathrm{Kg}$ were used in this study. Right and critical size bone defects (CSDs) were performed intraorally at the mandibular premolar area by the aid of trephine bur, of $6 \mathrm{~mm}$ outer diameter and $5 \mathrm{~mm}$ inner diameter. Right side bone defects (Experimental) were filled with mixture of ( $\beta$-TCP) and (BMSCs) which was taken from bone marrow aspirate concentrates (BMACs) from the same animal. Meanwhile, the left side bone defects (control) were left empty for healing spontaneously. The effect of (BMSCs) was assessed histologically as well as histomorphometrically after 6 weeks.

RESULTS Greater amount of bone formation was noticed in (CSDs) filled with ( $\beta$-TCP) and (BMSCs). The amounts of new bone formed in the defects filled with ( $\beta$-TCP) and (BMSCs) were significantly greater than those formed within the defects that were left empty. CONCLUSIONS: Autogenous (BMSCs) has excellent osteogenic characteristics and can be added to bone grafts following surgical and periodontal surgeries to enhance bone regenerative capacity.
\end{abstract}

KEYWORDS: Antifungal activity, Candida albicans, photocatalytic nano-titanium dioxide, silicone elastomer.

1. Assistant lecturer of Oral Biology - Faculty of Dentistry - Alexandria University.

2. Professor and Head of Oral Biology Department - Faculty of Dentistry - Alexandria University.

3. Professor of Oral Biology Department - Faculty of Dentistry - Alexandria University.

4. Professor of Clinical Pathology Department - Faculty of Medicine - Alexandria University.

\section{INTRODUCTION}

Achieving bone regeneration for bone defects is a crucial issue in oral and maxillofacial surgery. Many cases require bone regeneration, for example, reconstruction of bone defect after extirpation of jaw tumors and cysts, bone grafting for alveolar cleft, treatment of periodontal defects, at the extraction sites to prevent ridge resorption, for alveolar ridge reconstruction and preprosthetic surgery of sinus lifting for bone deficiency(1). Critical-size defect (CSD) is one of the most commonly used in vivo experimental models for evaluation of bone healing. (CSD) is defined as a defect that won't heal without intervention(2). Multiple methods have been studied for the restoration of bone defects in the field of craniomaxillofacial reconstructive surgery and orthopedic surgery. Historically, fresh autogenous bone grafting has been used to carry out this, but it is associated with complications including; infection, malformation, pain, and loss of function(3). Allografts represent the second most common bone-grafting technique, but they are associated with risks of immunoreactions, transmission of infections and they have reduced osteoinductive properties and no cellular component(4). The complications resulting from autograft and allograft harvests have inspired the development of alternative strategies for the repair of clinically significant bone defects. One alternative approach is the implantation of living cells that are directly osteogenic. According to tissue engineering concepts, it is possible to regenerate various tissues using living cells and an appropriate scaffold (5). Bone marrow has been shown to contain a population of stem cells capable of differentiating to the cells that form various tissues. These cells, referred to as mesenchymal stem cells (MSCs), are capable of forming bone when implanted ectopically in an appropriate scaffold(6). These cells represent two properties: self-renewal and multi-lineage differentiation, that render them an appropriate cell source for bone tissueengineering purposes (7).

Recently, (MSCs) have received widespread attention because of their potential utility in tissue engineering applications (8). As typical multipotent stem cells, (MSCs) have been shown to possess the capability to differentiate into a variety of cell types, including adipocytes, osteoblasts, chondrocytes, myoblasts and neuron-like cells(9). Mesenchymal stem cells can be isolated from bone marrow, periosteum, trabecular bone, adipose tissue, skeletal muscles and deciduous teeth (8). Bone marrow aspirate concentrates (BMACs) are a rich source of osteogenic stem cells and osteoconductive growth factors, ultimately used for the regeneration of bone defect. Specifically, (BMACs) contain a loaded mix of regenerative cells, including bone marrow derived mesenchymal stem cells (BMSCs), endothelial progenitor cells (EPCs), hematopoietic stem cells (HSCs), platelets, lymphocytes, and granulocytes. Each one of these components plays a critical role in the bone regeneration process (10). (BMSCs) have been proven to have proliferation and osteogenic differentiation capacities (1).

In vivo studies demonstrated that canine (BMSCs) has the potential to facilitate repair of bone defects, thus providing proof of concept for the clinical use of BMSCs 
for bone regeneration (11). The transplantation of (BMSCs) together with a suitable carrier is an essential prerequisite for in vivo bone formation (12). $\beta$-Tricalciumphosphate ( $\beta$ TCP) is a bioceramic material which has been extensively studied for its properties of high biocompatibility, relatively good biological absorbability and could be used as a matrix due to its excellent osteoconductive properties(13). Calcium phosphate scaffolds clearly support the osteogenic differentiation of human BMSCs and show ectopic bone formation that was originated from donor cells (14). It was found that ( $\beta$-TCP) loaded with (BMSCs) has excellent osteogenic characteristics and support its potential in tissue engineering to repair bone defects (15). It is confirmed that when ( $\beta$-TCP) is used as a scaffold for BMSCs, it acts as a raw material of regenerated cranial bone and that (BMSCs) have the potential to promote bone regeneration(16). To the best of our knowledge, there are few studies in the literature which investigated the effect of ( $\beta$-TCP) scaffold loaded with (BMSCs) taken from (BMACs) on bone defect healing and this is why the present investigation has been carried out. However, the null hypothesis of this study was assumed that no difference in bone defect regeneration will be obtained neither with the use of (BMSCs) nor without using it.

Aim of the study was to evaluate histologically and morphometrically the efficacy of autogenous (BMSCs) loaded in $\beta$-TCP graft in healing of critical-size mandibular defects.

\section{MATERIALS AND METHODS}

Animals \& experimental design: The ethical Committee of the Faculty of Dentistry, Alexandria University approved the protocol of this research. Ten male Mongrel dogs, each weighing $25 \mathrm{Kg}$, were used in this study. The animals were obtained from the Institute of Medical Research Alexandria University, with intact maxillary and mandibular teeth and showing good systemic and periodontal health. The animals were kept under the same nutritional and environmental conditions and received complete oral prophylaxis, antiparasitic treatment, multivitamins, and complete vaccines. A split mouth design was carried out in this study; the mandibular second premolars were selected for this study considering the right side as study group and the left side as control group.

Materials: $\beta$-TCP Synthetic Bone particles (AdbonePortugal) exhibiting up to $80 \%$ interconnected porosity with pore size $400 \mu \mathrm{m}$ and particle size ranging from 1 to $2 \mathrm{~mm}$, Ficoll separating medium (Sigma-Aldrich, St. Louis, MO, USA), Roswell Park Memorial Institute medium (RPMI) (ThermaFisher, Gibco, UK) and low-glucose Dulbecco's modified eagle medium (DMEM) (Lonza, Biowhittaker media, USA) were purchased to be used in this study.

\section{Surgical procedure:}

All operating procedures were performed under general anesthesia. Each animal was generally anaesthetized via intravenous injection of ketamine and xylaject (ADWIA Co, 10th of Ramadan City, Egypt), the dose of which was calculated on a basis of $30 \mathrm{mg} / \mathrm{kg}$ body weight.

Bone marrow aspirate concentrates (sampling and preparation):-

Five $\mathrm{ml}$ of bone marrow were aspirated 24 hours prior to implantation, from the iliac crest of each animal. The aspiration was performed under general anesthesia. Preservative free sodium heparin anticoagulant was used to prevent the aspirated bone marrow from coagulation. All preparation of (BMACs) was carried out under laminar air flow in the cell culture laboratory at the Institute of Medical Research Alexandria University. Dilution of bone marrow sample was performed with phosphate-buffered saline (PBS). Cells were gently layered on Ficoll separating solution (density, $1,077 \mathrm{~g} / \mathrm{ml}$ ). Centrifugation was done at 2000 rpm for 20 minutes.

The low density animal bone marrow mesnechymal stem cells-enriched mononuclear fraction was collected, washed by phosphate-buffered saline and centrifuged at $1200 \mathrm{rpm}$ for 10 minutes for 3 successive times. Cells were resuspended in (MSCs) complete culture media, lowglucose Dulbecco's modified eagle medium (with 4/5 g glucose and L-glutamine). B-TCP particles were hydrated with saline for $2 \mathrm{~min}$, and then the saline was removed and $(\beta$-TCP) particles were mixed with (BMSCs)-enriched mononuclear fraction washed by (PBS). The (BMSCs) / ( $\beta$ TCP) composites were then incubated for 24 hours to allow the cells to attach before implantation.

\section{Defect creation: -}

A $5 \mathrm{~cm}$ intraoral incision was made in the mandibular buccal sulcus at the premolar region in the right and left sides using Bard Parker scalpel handle no.3 and a disposable blade no.15. The mucooperiosteal flap was reflected using a periosteal elevator and bone was exposed through blunt dissection. In each dog two rounded critical sized bone defects $5 \mathrm{~mm}$ diameter and $5 \mathrm{~mm}$ depth, were created using trephine bur; one on the right side and the other on the left. The 10 critical sized bone defects on the right sides served as a study group and were implanted with ( $\beta$-TCP) / (BMSCs) composites. The osseous defects on the left side served as a control group and were left empty to undergo spontaneous healing. For both groups, the mucoperiosteal flaps were repositioned and stitched using 4/0 polyglycolic acid suture.

Euthanasia was done after 6 weeks by an overdose of the anesthetic solution Thiopentone Sodium injected rapidly. The mandibles were dissected out, sectioned into two halves and fixed in $10 \%$ neutral buffered formalin. After fixation, the mandibles were decalcified in 5\% trichloroacetic acid for 4 months. After decalcification, the tissue blocks containing the experimental regions were separated from the rest of the mandibles, washed, dehydrated in ascending order of ethanol and embedded in paraffin wax. Serial mesio-distal sections of $5 \mu \mathrm{m}$ thickness were cut and stained with Hematoxylin \& Eosin.

Histomorphometric analysis using Image J software was done to obtain the percentage of surface area of the formed bone compared to the total surface area of the created bone defect. Five sections of tissue from different standardized depths were used for quantification from each sample. A rectangle with standardized dimensions was drawn on the desired area to be measured using the image J program. The surface area of this selected region was measured by choosing Region of Interest (ROI) manager, and the measurement was recorded. The surface area occupied by the bone marrow spaces was selected using the wand tracing tool and the measurement was recorded.

The two recorded measurements were subtracted to obtain the surface area occupied only by bone, and its percentage to the total area selected was calculated. The measurements from three photographs were recorded and their mean was calculated for each of five sections obtained 
from each specimen. Statistical analysis of the obtained data was done using paired t-test to compare the percentage of new bone formed in the study and control groups after 6 weeks.

\section{RESULTS}

\section{Histological results:-}

Control group (defect was left empty):

The defect was filled with dense granulation tissue. Bone formation was restricted to one side of the defect wall with disruption of contact between the newly formed trabeculae and the native bone in some areas (Figure 1). The boundary of the defect showed osteoclastic activity (Figure 2). Some areas of the defect boundary showed deficiency in the bone formation with extension of the fibrous tissue aggregates towards these areas. Also the fibrous tissue was formed around the newly formed trabeculae that appeared confined to the lateral wall of the defect (Figure 2, 3).

Study group [defect was filled with (BMSCs) / $(\beta-$ TCP) composites]:

The defects were filled almost with dense compact bone which appeared to be of a high density and with different size of osteons (Figure 1). Extension of formation of the dense lamellated compact bone observed near to the center of the defect and consisted of numerous haversian systems enclosing rich blood supply within their haversian canals and volkman's canals (Figure 2). Another specimen taken from another animal demonstrated that the entire defect was filled with new bone in form of intercommunicating bone trabeculae enclosing bone marrow spaces of rich vascularity (Figure 3). The newly formed bone trabeculae appeared well-organized and mature with uniform thickness of trabeculae. The line of fusion was evident between the newly formed bone trabeculae and the native bone (Figure 3).

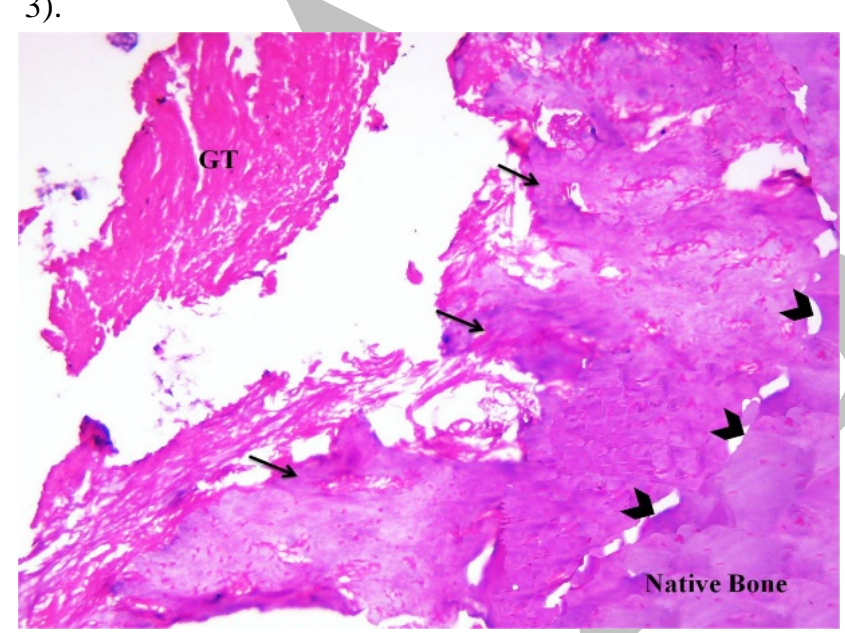

Figure 1: Light photomicrograph of the control group showing the bone formation appears restricted to one side of the defect wall (arrows). Note the dense aggregates of granulation tissue (GT) and the disruption of contact between the native and the

new bone trabeculae (arrowheads). H\&E stain; original magnification x100.

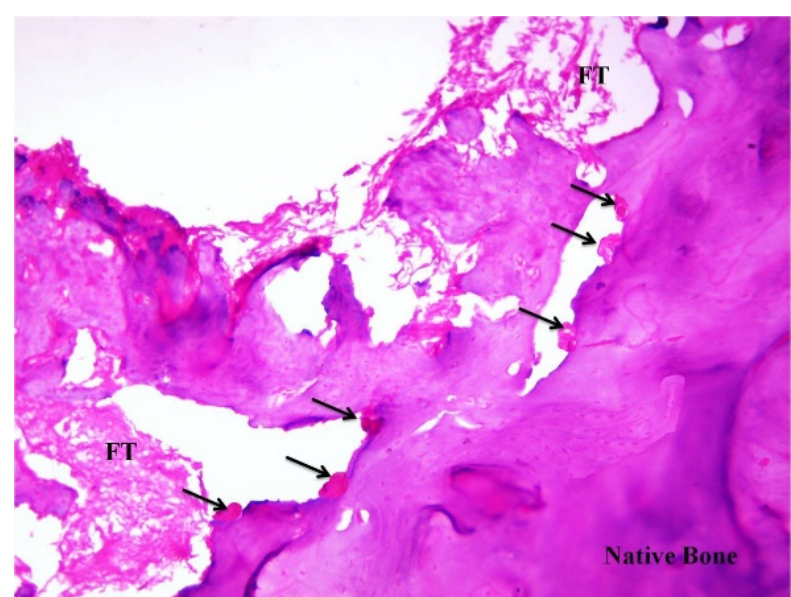

Figure 2: Light photomicrograph of the control group showing the boundary of the defect with osteoclastic activity (arrows) and the extension of fibrous tissue aggregates towards some areas at the defect boundary (FT). H\&E stain; original magnification x100.

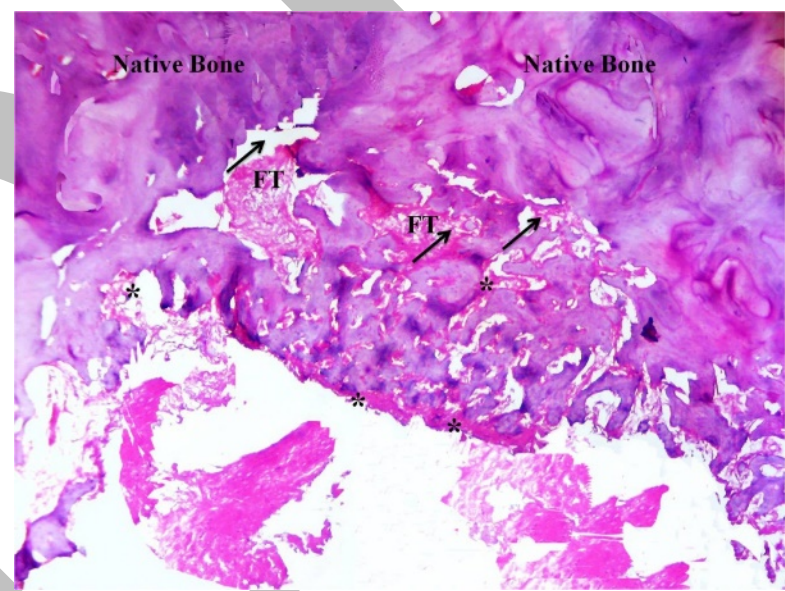

Figure 3: Light photomicrograph of the control group showing failure of bone formation in some areas of the defect boundary (arrows) and extension of fibrous tissue aggregates towards these areas (FT). Note the fibrous tissue that surround the newly formed bone trabeculae (asterisks) H\&E stain; original magnification $\mathrm{x} 40$.

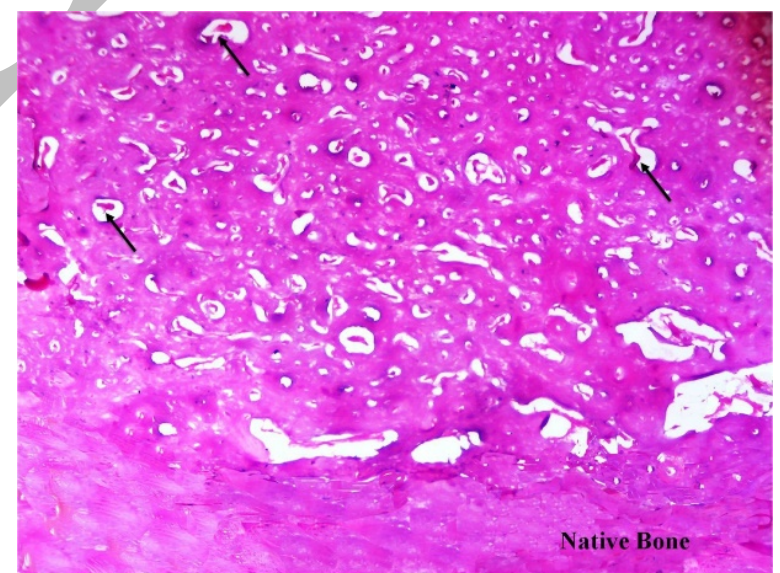

Figure 4: Light photomicrograph of the study group showing the dense appearance of the formed bone with limited figures of bone marrow spaces and the formation of different size osteons. Note the rich vascularity of the haversian canals (arrows). H\&E stain; original magnification $\mathrm{x} 40$ 


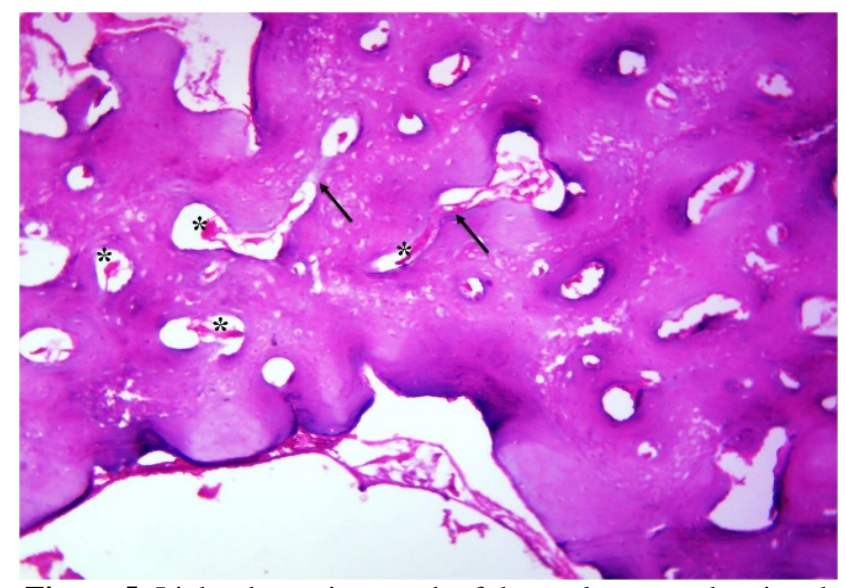

Figure 5: Light photomicrograph of the study group showing the continuation of bone formation in form of dense compact bone toward the center of the defect. Note the haversian and volkman's canals (arrows) with their profound vascularity (asterisks) H\&E stain; original magnification $\mathrm{x} 10$

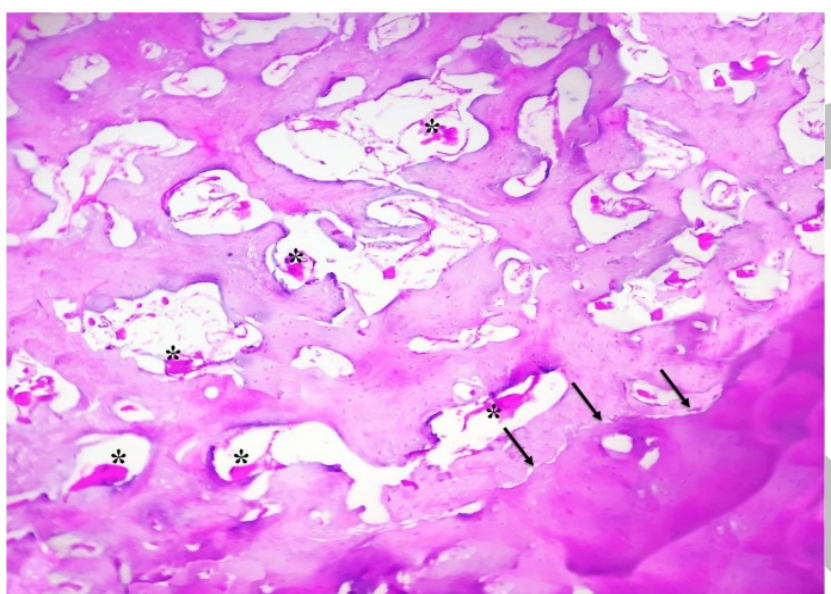

Figure 6: Light photomicrograph of the study group showing the organization and density of the formed bone trabeculae adjacent to the defect boundary. Note their thickness and the interconnectivity and the rich vascularity of the connective tissue intervening between these trabeculae (asterisks). Note the fusion line between the newly formed and the native bone (arrows) H\&E stain; original magnification x100.

\section{II-Histomorphometric analysis:-}

The mean values of the percentages of bone surface area formed during the healing of the bone defect in both control and study groups after 6 weeks were calculated and demonstrated in table (1).

Table 1: percentage of the newly formed bone occupying the defect area.

\begin{tabular}{|l|l|l|}
\hline Control & Study \\
\hline $35.00663 \%$ & $73.8762 \%$ \\
\hline $21.89194 \%$ & $52.96951 \%$ \\
\hline $29.77558 \%$ & $60.57466 \%$ \\
\hline $36.90384 \%$ & $73.24831 \%$ & \\
\hline $42.73529 \%$ & $64.41289 \%$ & \\
\hline $58.20495 \%$ & $53.26871 \%$ & P value \\
\hline $43.61536 \%$ & $64.33839 \%$ & $0.002 *$ \\
\hline Mean & Mean & \\
\hline $38.3048 \pm$ & $63.24124 \pm$ & \\
\hline
\end{tabular}

The percentage of new bone formed (NB \%) is defined as the percentage of bone formed in the region of interest (ROI). At 6 weeks, the mean NB\% for the control group was $38.3048 \%$ and $63.24124 \%$ for the study group (p value $=0.002)$. Therefore, the study group [defect was filled with (BMSCs) / ( $\beta$-TCP) composites] exhibited statistically significantly greater NB\% compared with the control group.

\section{DISCUSSION}

Bone marrow aspirate concentrates (BMACs) are commonly harvested from the patient's iliac crest to yield a rich source of osteogenic stem cells and osteoconductive growth factors, ultimately to repair and regenerate the bone defect (10). Over the years, (BMACs) have proved to be a safe alternative method to promoting bone unions, with effectiveness similar to autologous bone grafting. The most favorable features of bone marrow aspirate in terms of clinical application are its accessibility with little morbidity and the abundance of the cells. However bone marrow aspirates contain many osteoinductive growth factors as well as BMSCs, but are lacking an osteoconductive scaffold (17). Nowadays, it is argued that the bone marrow aspirates contains sufficient number and quality of (BMSCs) and have been shown to be effective in the treatment of bone defects, including tibial nonunions and osteonecrosis of the femoral head (10). Bone marrow derived mesenchymal stem cells (BMSCs) have been proven to have proliferation and osteogenic differentiation capacities $(1,8)$.

This study was performed in order to evaluate the effect of BMSCs on the bone formation when it was taken from bone marrow aspirate concentrates and mixed with $\beta$ TCP particles. - $\beta$-TCP bone graft was the material of choice to creates a resorbable interlocking network within the defect site to promote healing because of its resorbability and it has a good osteoconductive capacity due to its high porosity (up to $70 \%$ ) and inter-pore connectivity that can provide intercellular communication among osteocytes and (BMSCs) (18). $\beta$-TCP is known to be biocompatible, osteoconductive, and biodegradable, but not osteopromotive (19). Our finding regarding BMSCs/ B-TCP composite and its effect on new bone formation in critical size osseous defects showed that this composite notably promote bone formation and the defect was filled almost entirely with mature trabecular bone that containing mature bone marrow zones within 6 weeks. The new bone formed in the control groups was lower than that found in the study group.

The new bone formed in the study group was higher and bone trabeculae were also thicker. These results are in agreement with the findings of Brennan et al (20) who also examined the effect of (BMSCs) and calcium phosphate scaffold, but in critical size calvarial bone defects of nude mice. In another study performed by Boo et al (6) stated that ( $\beta$-TCP) loaded with (BMSCs) has excellent osteogenic characteristics and support its potential in tissue engineering to repair bone defects. Moreover, in a recent study that was performed to treat surgically created bone defects in the facial skeleton with individually prepared absorbable biocomposites that involve a combination of a calcium phosphate biomaterial and autologous bone marrow, autologous bone marrow was found to enhance bone growth within the biocomposites(17). 
As mentioned previously that the bone marrow aspirates contains sufficient number and quality of BMSCs and according to previous studies $(1,8)$ (BMSCs)-based bone augmentation procedures induced higher levels of bone formation compared to acellular treatments which support our finding. The mode of action of the enhanced bone healing is apparently due to that BMSCs exhibit functional, tissue-specific biodiversity mediated by direct "cell to- cell" cross talk associated with adhesion molecules activity, cytokines, growth factors and cell signaling pathways(12).

Another interesting finding was the high vascularity to the defect area in the study group compared to the control. This could be explained by Bose et al(10) who found that BMACs contain a loaded mix of regenerative cells, including BMSCs, endothelial progenitor cells (EPCs), hematopoietic stem cells (HSCs), platelets, lymphocytes, and granulocytes. Each one of these components plays a critical role in the bone regeneration process. The granulocytes release vascular endothelial growth factor (VEGF) which induces the angiogenesis to promote the healing process (10). This positive action of BMSCs on bone formation during the healing of osseous defects suggest that the direct delivery of BMSCs promotes osteoinductive bone formation, thus it would be promising as a method of achieving rapid and predictable bone regeneration of osseous defects in periodontal disease.

\section{CONCLUSION}

The direct application of BMSCs combined with porous $\beta$ TCP particles in the osseous defect may be effective for achieving favorable new bone formation in large osseous defects. Such approach is expected to provide improved clinical outcome in the effort to restore large osseous defects in which osteoconductive bone substitutes have only limited bone healing capacity.

\section{CONFLICT OF INTEREST}

The authors declare that they have no conflicts of interest.

\section{REFERENCES}

1.Shayesteh YS, Khojasteh A, Soleimani M, Alikhasi M, Khoshzaban A, Ahmadbeigi N. Sinus augmentation using human mesenchymal stem cells loaded into a $\beta$-tricalcium phosphate/hydroxyapatite scaffold. Oral Surg Oral Med Oral Pathol Oral Radiol Endod. 2008; 106(2):203-9.

2. Poser L, Matthys R, Schawalder P, Pearce S, Alini M, Zeiter S. A standardized critical size defect model in normal and osteoporotic rats to evaluate bone tissue engineered constructs. Biomed Res Int. 2014; 2014.

3. Suenaga H, Furukawa KS, Suzuki Y, Takato T, Ushida T. Bone regeneration in calvarial defects in a rat model by implantation of human bone marrow-derived mesenchymal stromal cell spheroids. J Mater Sci Mater Med. 2015; 26(11):1-9.

4. Delloye C, Cornu O, Druez V, Barbier O. Bone allografts. J Bone Jt Infect. 2007;89(5):574-80.

5. Haynesworth S, Goshima J, Goldberg V, Caplan A. Characterization of cells with osteogenic potential from human marrow. Bone. 1992; 13(1):81-8.

6. Boo JS, Yamada Y, Okazaki Y, Hibino Y, Okada K, Hata $\mathrm{K}-\mathrm{I}$, et al. Tissue-engineered bone using mesenchymal stem cells and a biodegradable scaffold. J Craniofac Surg. 2002;13(2):231-9.

7. Heng BC, Cao T, Stanton LW, Robson P, Olsen B. Strategies for directing the differentiation of stem cells into the osteogenic lineage in vitro. J Bone Miner Res. 2004; 19(9):1379-94.

8. Vahabi S, Amirizadeh N, Shokrgozar M, Mofeed R, Mashhadi A, Aghaloo M, et al. A comparison between the efficacy of Bio-Oss, hydroxyapatite tricalcium phosphate and combination of mesenchymal stem cells in inducing bone regeneration. Chang Gung Med J. 2011; 35(1):28-37.

9. Wei X, Yang X, Han Z-p, Qu F-f, Shao L, Shi Y-f. Mesenchymal stem cells: a new trend for cell therapy. Acta Pharmacol Sin. 2013;34(6):747-54.

10. Bose S, Roy M, Bandyopadhyay A. Recent advances in bone tissue engineering scaffolds. Trends Biotechnol. 2012;30(10):546-54.

11. Arinzeh TL, Peter SJ, Archambault MP, Van Den Bos C, Gordon S, Kraus K, et al. Allogeneic mesenchymal stem cells regenerate bone in a critical-sized canine segmental defect. J Bone Joint Surg Am. 2003;85(10):1927-35.

12. Šponer P, Kučera T, Diaz-Garcia D, Filip S. The role of mesenchymal stem cells in bone repair and regeneration. Eur J Orthop Surg Traumatol. 2014;24(3):257-62.

13. Yang L, Sun H-Y, Qi N-M, Tan W-S. The effects of $\beta$ tricalcium phosphate 3D scaffold in-situ cryopreservation on the migration rate and osteogenic ability of mesenchymal stem cells. Bioresour Bioprocess. 2012;17(1):195-202.

14. Matsushima A, Kotobuki N, Tadokoro M, Kawate K, Yajima H, Takakura Y, et al. In Vivo Osteogenic Capability of Human Mesenchymal Cells Cultured on Hydroxyapatite and on $\beta$-Tricalcium Phosphate. Artif Organs. 2009; 33(6):474-81.

15. Umeda H, Kanemaru Si, Yamashita M, Kishimoto M, Tamura Y, Nakamura T, et al. Bone Regeneration of Canine Skull Using Bone Marrow-Derived Stromal Cells and $\beta$ Tricalcium Phosphate. Laryngoscope. 2007; 117(6):9971003.

16. Warren SM, Nacamuli RK, Song HM, Longaker MT. Tissue-engineered bone using mesenchymal stem cells and a biodegradable scaffold. J Craniofac Surg. 2004;15(1):347.

17. Russmueller G, Moser D, Spassova E, Plasenzotti R, Poeschl P, Seemann R, et al. Tricalcium phosphate-based biocomposites for mandibular bone regeneration-A histological study in sheep. J Craniomaxillofac Surg. 2015;43(5):696-704.

18. Nakagawa Y, Muneta T, Tsuji K, Ichinose S, Hakamatsuka $\mathrm{Y}$, Koga $\mathrm{H}$, et al. $\beta$-Tricalcium phosphate micron particles enhance calcification of human mesenchymal stem cells in vitro. J Nanomater. 2013; 2013:5.

19. Wang XL, Xie XH, Zhang G, Chen SH, Yao D, He K, et al. Exogenous phytoestrogenic molecule icaritin incorporated into a porous scaffold for enhancing bone defect repair. J Orthop Res. 2013; 31(1):164-72.

20.Brennan MÁ, Renaud A, Amiaud J, Rojewski MT, Schrezenmeier H, Heymann D, et al. Pre-clinical studies of bone regeneration with human bone marrow stromal cells and biphasic calcium phosphate. Stem Cell Res Ther. 2014; 5(5):1. 